

\title{
A New Symmetry for the Packing of Amphiphilic Direct Micelles \\ Marianne Clerc
}

\section{To cite this version:}

Marianne Clerc. A New Symmetry for the Packing of Amphiphilic Direct Micelles. Journal de Physique II, 1996, 6 (7), pp.961-968. 10.1051/jp2:1996110 . jpa-00248352

\section{HAL Id: jpa-00248352 https://hal.science/jpa-00248352}

Submitted on 1 Jan 1996

HAL is a multi-disciplinary open access archive for the deposit and dissemination of scientific research documents, whether they are published or not. The documents may come from teaching and research institutions in France or abroad, or from public or private research centers.
L'archive ouverte pluridisciplinaire HAL, est destinée au dépôt et à la diffusion de documents scientifiques de niveau recherche, publiés ou non, émanant des établissements d'enseignement et de recherche français ou étrangers, des laboratoires publics ou privés. 


\title{
A New Symmetry for the Packing of Amphiphilic Direct Micelles
}

\author{
Marianne Clerc $\left(^{*}\right)$ \\ Laboratoire de Physique des Solides, Bât 510, Université Paris-Sud, 91405 Orsay Cedex, France
}

(Received 30 October 1995, revised 13 March 1996, accepted 25 April 1996)

PACS.61.10.-i - X-ray diffraction and scattering
$\begin{aligned} \text { PACS.61.30.Eb - } & \text { Experimental determination of smectic, nematic, cholesteric, } \\ & \text { and other structures }\end{aligned}$

\begin{abstract}
Thanks to X-ray scattering, we have studied the $\mathrm{C}_{12} \mathrm{EO}_{8}$ /water binary system in the region located between the isotropic phase and the direct hexagonal mesophase. We have discovered two 3D-periodic phases in this region: one with a cubic symmetry (space group $\mathrm{Pm} \overline{3} \mathrm{n}$ ), the other with a hexagonal symmetry (space group $\mathrm{P} 6_{3} / \mathrm{mmc}$ ). Both phases consist in a regular packing of direct micelles build with the surfactant molecules in water. The cubic phase have already been observed in other lyotropic systems. On the other hand, the hexagonal phase is a new type of structure for such systems. We assume for this phase a hexagonal compact structure.
\end{abstract}

Résumé. - Nous avons étudié par diffraction des rayons $\mathrm{X}$ la région du système binaire $\mathrm{C}_{12} \mathrm{EO}_{8}$ /eau comprise entre la phase isotrope et la phase hexagonale directe. Nous y avons mis en évidence deux phases périodiques tridimensionnelles : l'une avec une symétrie cubique (groupe d'espace $\mathrm{Pm} \overline{3} \mathrm{n}$ ), l'autre avec une symétrie hexagonale (groupe d'espace $\mathrm{P} 6_{3} / \mathrm{mmc}$ ). Ces deux phases résultent de l'arrangement périodique de micelles directes, formées par les molécules de surfactant dans l'eau. Ce type de phase cubique micellaire a déjà été observé dans d'autres systèmes lyotropes. Par contre, une telle phase hexagonale n'a jamais été observée précédemment. Nous proposons pour cette phase une structure hexagonale compacte.

\section{Introduction}

It is well known that micellar cubic phases appear in lyotropic systems $[1,2]$. They consist in a 3D regular arrangement either of direct micelles, formed by amphiphilic molecules in water, or of inverse micelles of water embedded in a continuous amphiphilic matrix. These phases have been studied thanks to NMR, X-ray scattering and electronic microscopy [1,2]. Two main space groups have been observed: $\operatorname{Pm} \overline{3} \mathrm{n}$ and $\mathrm{Fd} \overline{\mathrm{3}} \mathrm{m}$. Models for these two space groups have been established. They all involve two different species of micelles inside the structure. Some geometrical arguments have explained why these two types of cubic arrangements are favourable for the packing of the micelles [3].

Recently, other cubic space groups for direct micellar phases have been observed in the ganglioside/water systems [4]. It was a clue that structures with space groups $\mathrm{Pm} \overline{3} \mathrm{n}$ and

$\left({ }^{*}\right)$ e-mail: clerc@lps.u-psud.fr

(C) Les Éditions de Physique 1996 
Fd $\overline{3} \mathrm{~m}$ are not the only possible ones. So the polymorphism of micellar phases had to be investigated deeper. In this paper, we display another periodic arrangement of direct micelles, this one having a new feature: a hexagonal symmetry!

The existence of a micellar cubic region in the binary mixture $\mathrm{C}_{12} \mathrm{EO}_{8}$ (octa-ethylene glycol mono n-dodecyl ether) $/ \mathrm{H}_{2} \mathrm{O}$ was first reported in [5]. Our aim was to study the structure of this phase, to get a new set of experimental data, in order to improve the description of the micellar cubic phases. Our first surprise during these experiments was that there are two phases instead of one in the region indicated in [5]. If we look at the phase diagram of the $\mathrm{C}_{14} \mathrm{EO}_{8} / \mathrm{H}_{2} \mathrm{O}$ mixture [6], a very similar system, two distinct micellar cubic phases are also identified, unfortunately without any structural data available. Which strengthens our result.

Going further into our X-ray investigations, our second surprise was that one of the two phases has not a cubic symmetry, but a hexagonal one. It took us a long time to realize it, because, as far as we knew, a triply-periodic micellar phase was always cubic. In spite of numerous efforts, finding some cubic indexations of the powder patterns proved to be a failure. Fortunately, the single-crystals analysis gave an indisputable evidence of a hexagonal symmetry. Every X-ray pattern could then be easily interpreted; we will show it in Section 3.

\section{Materials and Methods}

$\mathrm{C}_{12} \mathrm{EO}_{8}$ was purchased from Nikko Chemical [7] and used without further purification. Deuterated water was mixed with the surfactant and equilibrated for a few weeks at room temperature. Concentrations between $30 \%$ and $43 \%\left(\mathrm{w} / \mathrm{w}\right.$ ) of $\mathrm{C}_{12} \mathrm{EO}_{8}$ in $\mathrm{D}_{2} \mathrm{O}$ were used.

The mixtures were observed during the cooling from the room temperature down to $8{ }^{\circ} \mathrm{C}$. First observations of the samples by optical microscopy were done between crossed polarizers. For the X-ray experiments the samples were placed in Lindeman cylindrical glass capillaries $(\varnothing 1 \mathrm{~mm})$. The X-ray apparatus used for the single-crystals analysis was a Rigaku rotating anode generator (copper wavelength $\lambda=1.54 \AA$ ). The powders analysis were made on the synchrotron beam line D 43 at the LURE laboratory. Photographic films and image plates have been used as detection.

The best results concerning the single-crystals analysis were obtained with samples equilibrated in a refrigerator $\left(8^{\circ} \mathrm{C}\right)$ for several months.

\section{Results}

3.1. Qualitative Phase Diagram (Fig. 1). - At room temperature, the samples are fluid and isotropic for concentrations up to about $39 \%(\mathrm{w} / \mathrm{w})$. Beyond $39 \%$, the samples become viscous; the texture of the direct hexagonal mesophase is clearly identified through optical microscopy. By cooling the samples which were under $39 \%$, we observe a highly viscous isotropic phase (Fig. 1). For samples of concentration above 39\%, a biphasic region between the hexagonal phase and a highly viscous isotropic phase is abserved. Going on cooling, there remains only the isotropic phase.

3.2. X-Ray Data: Powder Diffraction. - We wanted to determine the structure of the high viscous isotropic phase appearing at low temperature in our samples thanks to X-ray scattering. Two different kinds of X-ray patterns were obtained, depending on the concentration.

3.2.1. Concentration above 39\%. - Several high-resolved powder diffraction patterns were obtained on the synchrotron beam line D 43 at the LURE laboratory. The diffraction rings are easily interpreted using a cubic unit cell, as detailed in Table I. A unit cell of parameter 




Fig. 1. - Qualitative phase diagram of the binary mixture $\mathrm{C}_{12} \mathrm{EO}_{8}$ in $\mathrm{D}_{2} \mathrm{O}$. The transition lines we determined during our study are drawn in heavy black lines. They correspond to changes in the viscosity and/or in the optical aspect between crossed polarizers of the samples. The dashed fine lines are extrapolated from the phase diagram of the $\mathrm{C}_{14} \mathrm{EO}_{8}$ /water system [6] and do not correspond to observations. Especially, we do not crossed experimentally the coexistence region of the two micellar phases, which is probably narrow versus concentration. The vertical lines correspond to the samples used for the $\mathrm{X}$-ray scattering analysis. $\mathrm{H}_{\alpha}$ indicates the hexagonal direct phase, $\mathrm{Q}_{\mathrm{m}}$ the cubic micellar phase and $\mathrm{H}_{\mathrm{m}}$ the hexagonal micellar phase.

$a=120.26 \pm 0.19 \AA$ is measured. The observed reflections are compatible with the space group $\operatorname{Pm} \overline{3} \mathrm{n}$, usually observed for micellar direct cubic phases (see Sect. 1). The unit cell of this structure contains six ellipsoidal micelles and two quasi-spherical micelles [1-3]. We will see in Section 3.4 that this model with eight micelles per unit cell is compatible with the physico-chemical data. So we admit the space group $\mathrm{Pm} \overline{3} \mathrm{n}$ for this phase. We did measure the parameter of the direct hexagonal phase in coexistence with this cubic phase: $a_{\mathrm{H}_{\alpha}}=$ $59.40 \pm 0.14 \AA$ (Tab. I). No epitaxial relations between inter-reticular distances are observed between these two phases.

3.2.2. Concentration below 39\%. - We manage to obtain several similar powder diffraction patterns in the range $34.3-38 \%(\mathrm{w} / \mathrm{w})$. As stressed in the introduction (Sect. 1), we performed many unsuccessful indexations of these diagrams, assuming a cubic unit cell.

We also began to perform single-crystal diffraction patterns in the same concentration range. The interpretation of the reciprocal planes, still assuming a cubic unit cell, happen to be impossible.

As specified below, the single-crystal diffraction patterns could be interpreted by assuming a hexagonal unit cell. Coming back to the powder diffraction patterns, we found accurate indexations for them, assuming a hexagonal unit cell. Results are given in Table II. At $38 \%$ $(\mathrm{w} / \mathrm{w}$ ), we measure a hexagonal parameter $a=68.81 \pm 0.14 \AA$, and a ratio $R=c / a=1.623$. Extinctions are compatible with the hcp structure factor, as detailed below.

3.3. X-Ray Data: Single-Crystal Analysis below 39\%. - We focused on the structure of the micellar phase below $39 \%$, for which the assumption of a cubic unit cell did not give satisfactory results. We succeeded in growing sufficiently large single-crystals $\left(\sim 1 \mathrm{~mm}^{3}\right)$ in the Lindeman capillaries. In order to perform their analysis, we fixed them on a goniometric 
Table I. - Powder diffraction data of the cubic phase $\left(\mathrm{Q}_{\mathrm{m}}\right)$ and of the direct hexagonal mesophase $\left(\mathrm{H}_{\alpha}\right)$. Results are given for a sample of concentration $40 \%(w / w)$, located in the biphasic region $\left(\mathrm{Q}_{\mathrm{m}} / \mathrm{H}_{\alpha}\right)$. The parameter of the cubic phase is equal to $a=120.26 \pm 0.19 \AA$ and the parameter of the hexagonal phase is equal to $a_{\mathrm{H}_{\alpha}}=59.40 \pm 0.14 \AA$. The observed reflections for the cubuc phase (values of $s^{2}=h^{2}+k^{2}+l^{2}$ ) are compatible with the Pm $\overline{3} n$ space group.

\begin{tabular}{||c|c|c||}
\hline \hline$d_{h k l}(\AA)$ & $s^{2}$ & $a(\AA)=d_{h k l} s$ \\
\hline 60.17 & 4 & 120.34 \\
53.91 & 5 & 120.55 \\
49.17 & 6 & 120.44 \\
42.41 & 8 & 119.95 \\
34.74 & 12 & 120.34 \\
33.16 & 13 & $(119.56)$ \\
32.08 & 14 & 120.03 \\
30.08 & 16 & 120.32 \\
29.18 & 17 & 120.31 \\
26.84 & 20 & 120.03 \\
\hline$d_{h k l}(\AA)$ & $s^{2}$ & $d_{100}(\AA)=d_{h k l} s$ \\
\hline 51.43 & 1 & 51.43 \\
29.71 & 3 & 51.46 \\
25.72 & 4 & 51.44 \\
\hline
\end{tabular}

$40 \%(\mathrm{w} / \mathrm{w})$

Cubic phase $(\mathrm{Pm} \overline{3} \mathrm{n}): a=120.26 \pm 0.19 \AA$

$\mathrm{H}_{\alpha}$ phase: $a_{\mathrm{H}_{\alpha}}=59.40 \pm 0.14 \AA$

head, movable around its vertical axis. Thus, all possible orientations of the single-crystals have been observed.

In pattern (a) of Figure 2, the X-ray beam is parallel to a symmetry axis of a single-crystal. Three types of reflection $((1, \overline{1}, 0,0),(2, \overline{1}, \overline{1}, 0)$ and $(2, \overline{2}, 0,0)$ in the hexagonal four indices notation) form a figure of diffraction with a six-fold symmetry. Starting from this position, we rotated continuously the sample around the vertical axis over $360^{\circ}$. Making the hypothesis of a cubic symmetry, the starting position of pattern (a) would correspond to the X-ray beam being parallel to a three-fold axis. As we know that four equivalent axes of three-fold symmetry exist in the case of a cubic symmetry, we expected to observe during the rotation the appearance of equivalent Bragg reflections to the ones observed on pattern (a). It never happened: all the reflections of pattern (a), excepting the ones located on the rotation axis, are observed only at the starting position. As a conclusion, the symmetry axis of pattern (a) is unique, and the symmetry is not cubic.

We regard this symmetry axis as the $c^{*}$ axis of a hexagonal symmetry. In Figure 2, three reciprocal planes are shown, and their orientations respecting to the $c^{*}$ axis are given. Then the indexation of the Bragg spots is obtained. Eventually, the whole reciprocal space has been reconstructed. We then observe some extinctions, for instance the $(0,0,0,1)$ and $(0,0,0,3)$ 




a)

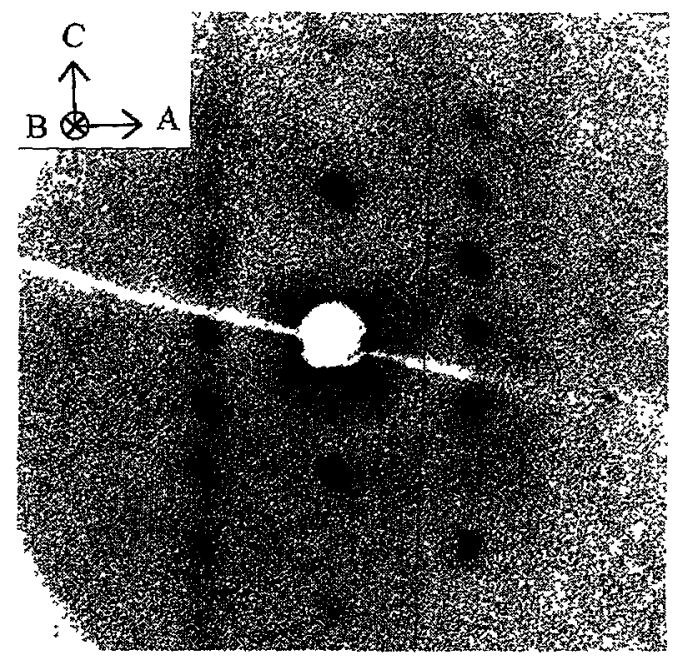

c)

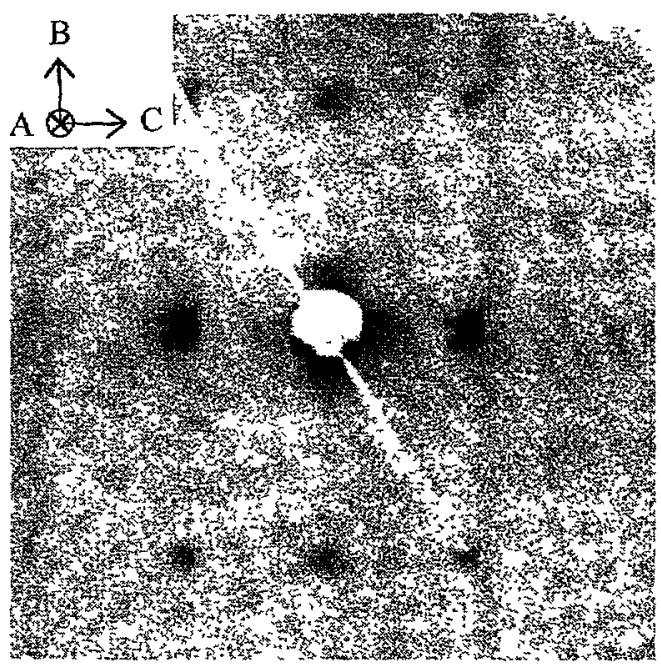

b)

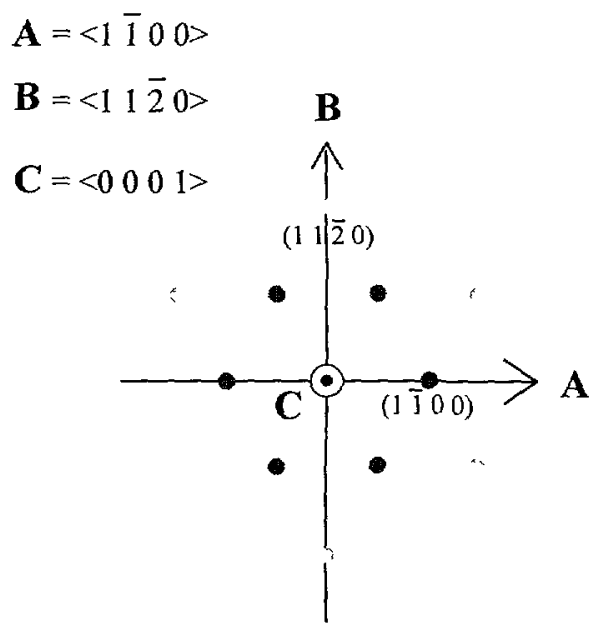

d)

Fig. 2. - Single-crystal diffraction patterns of the hexagonal micellar phase. Three diffraction patterns obtained on image-plates are reproduced. The four indices hexagonal notation is used in this caption. a) X-ray beam along the $c^{*}$ axis (exposure time of four hours): the $(1, \overline{1}, 0,0),(2, \overline{1}, \overline{1}, 0)$ and $(2, \overline{2}, 0,0)$ reflections, plus the reflections obtained by the circular permutations of their first three indices form a six-fold symmetric pattern. b) X-ray beam along the $\langle 1, \overline{1}, 0,0\rangle$ reciprocal direction (exposure time of three hours): the $(0,0,0,2)$ reflection (along the $c^{*}$ axis), the $(1,1, \overline{2}, 0)$ and the $(1,1, \overline{2}, 2)$ are located on a rectangular $2 \mathrm{D}$ array of reflections. c) X-ray beam along the $\langle 1,1, \overline{2}, 0\rangle$ reciprocal direction (exposure time of three hours): one observes the $(0,0,0,2)$ and the $(0,0,0,4)$ reflections along the $c^{*}$ axis. The $(1, \overline{1}, 0,0),(1, \overline{1}, 0,1),(1, \overline{1}, 0,2)$ and $(1, \overline{1}, 0,3)$ reflections are aligned in a row parallel to the $c^{*}$ axis. d) Relative orientations of the different $X$-ray patterns in the reciprocal space. 
Table II. - Powder diffraction data of the hexagonal phase. Indexation of the reflections assuming a hexagonal unit cell is given for two samples of concentrations $38 \%$ and $34.3 \%$ ( $w / w)$. hkil are the indices in the four indices notation and $H K l$ are the indices in the three indices notation. $a$ and $c$ are the two unit cell parameters and $R$ is equal to the ratio $c / a$. We use the following relation: $\frac{1}{d_{H K l}^{2}}=\frac{1}{d_{100}^{2}}\left(H^{2}+K^{2}+H K\right)+\frac{l^{2}}{c^{2}}=\frac{1}{a^{2}}\left(\frac{4}{3}\left(H^{2}+K^{2}+H K\right)+\frac{1}{R^{2}} l^{2}\right)=$ $\frac{s^{2}}{a^{2}}$. All the refiections are compatible with the structure factor of the hccp (3.3). Taking a value of $R$, we derive a value of a for all different reflections and calculate the average value of $a$ and the corresponding statistical error. The best value of $R$ is determined by minimizing this statistical error. Following this criterion, the best value of $R$ and the associated average value of a are given for the two samples. The statistical error on $a$ is about $0.15 \AA$ for the two samples, and is of the same order of magnitude as for the cubic phase indexation (Tab. I). The value of the unit cell volume is greater for the sample of concentration $34.3 \%(w / w)$ than for the sample of concentration $38 \%(w / w)$. It is in agreement with our model assuming a constant volume of a micelle and a greater water volume fraction at $34.3 \%(w / w)$ than at $38 \%(w / w)$.

\begin{tabular}{|c|c|c|c|c|c|c|c|c|c|}
\hline$h$ & $k$ & $i$ & $l$ & $H K l$ & $s^{2}=\frac{4}{3}\left(H^{2}+K^{2}+H K\right)+\frac{1}{R^{2}} l^{2}$ & $\begin{array}{c}d_{H K l} \\
(\AA)\end{array}$ & $a(\AA)$ & $\begin{array}{c}d_{H K l} \\
(\AA)\end{array}$ & $a(\AA)$ \\
\hline 1 & -1 & 0 & 0 & 100 & $4 / 3$ & 59.57 & 68.79 & 60.97 & 70.40 \\
\hline 0 & 0 & 0 & 2 & 002 & $4 / R^{2}$ & 55.92 & 68.91 & - & - \\
\hline 1 & -1 & 0 & 1 & 101 & $4 / 3+1 / R^{2}$ & 52.81 & 69.12 & 53.81 & 70.35 \\
\hline 1 & -1 & 0 & 2 & 102 & $4 / 3+4 / R^{2}$ & 40.74 & 68.80 & 41.69 & 70.22 \\
\hline 2 & -1 & -1 & 0 & 110 & 4 & 34.36 & 68.72 & 35.07 & 70.14 \\
\hline 1 & -1 & 0 & 3 & 103 & $4 / 3+9 / R^{2}$ & 31.47 & 68.59 & 32.27 & 70.08 \\
\hline 2 & -2 & 0 & 0 & 200 & $16 / 3$ & 29.82 & 68.87 & 30.34 & 70.07 \\
\hline 2 & -1 & -1 & 2 & 112 & $4+4 / R^{2}$ & 29.29 & 68.81 & 30.06 & 70.52 \\
\hline 2 & -2 & 0 & 1 & 201 & $16 / 3+1 / R^{2}$ & 28.73 & 68.67 & 29.38 & 70.20 \\
\hline
\end{tabular}

reflections (Fig. 2). As a rule, here are the general conditions of extinction:

$(h k i l)=(h h 2 \bar{h} 2 n+1) \quad$ (four indices notation).

We propose a hexagonal compact structure for this phase, with a unit cell containing two identical micelles at positions $(1 / 3,2 / 3,1 / 4)$ and $(2 / 3,1 / 3,3 / 4)$. The structure factor is equal to (three indices notation):

$$
F_{H K l}=\mathrm{e}^{-2 \imath \pi\left(\frac{H+2 K}{3}+\frac{l}{4}\right)}+\mathrm{e}^{-2 \imath \pi\left(\frac{2 H+K}{3}+\frac{3 l}{4}\right)}=\mathrm{e}^{-2 \imath \pi\left(\frac{H+2 K}{3}+\frac{l}{4}\right)}\left(1+(-1)^{l} \mathrm{e}^{-2 \imath \pi\left(\frac{H-K}{3}\right)}\right)
$$

Using the four indices notation, we obtain the following values for the intensity derived from this structure factor:

$$
\begin{aligned}
& l=2 n \quad \text { if } \quad h k i=h h 2 \bar{h} \text { then }\left|F_{h k l l}\right|^{2}=4 \text { else }\left|F_{h k l l}\right|^{2}=1 \\
& l=2 n+1 \quad \text { if } \quad h k i=h h 2 \bar{h} \text { then }\left|F_{h k l l}\right|^{2}=0 \text { else }\left|F_{h k \imath l}\right|^{2}=3
\end{aligned}
$$

This is consistent with the observed extinctions. The space group of this structure is: $\mathrm{P} 6_{3} / \mathrm{mmc}$. Notice that this space group is not uniquely determined by the observed extinctions (two 
other hexagonal space groups are compatible with the extinctions) but by the assumption of a hexagonal compact structure. In the following section, we will show that the structure we propose with two micelles per hexagonal unit cell is also consistent with the physico-chemical data.

3.4. Aggregation Number of the Micelles. - A crude estimation of the aggregation number of the micelles in both micellar phases will be now discussed.

We can simply estimate the density of the binary mixture by ignoring solvatation effects on the molecular volumes. We know the densities of $\mathrm{D}_{2} \mathrm{O}: 20 / 18 \mathrm{~g} \mathrm{~cm}^{-3}$ and of $\mathrm{C}_{12} \mathrm{EO}_{8}$ [7]: $0.99 \mathrm{~g} \mathrm{~cm}^{-3}$. No corrections versus temperature are included for this crude estimate. We will take for the density $d$ of a binary mixture of weight fraction $x$ in $\mathrm{C}_{12} \mathrm{EO}_{8}$ :

$$
\frac{1}{d}=\frac{1}{100}\left(\frac{x}{0.99}+\frac{100-x}{(20 / 18)}\right) .
$$

We calculate for the sample of concentration $38 \%$ a density of $1.062 \mathrm{~g} \mathrm{~cm}^{-3}$. For this sample, the volume of the hexagonal unit cell is equal to: $V_{\text {u.c. }}=\frac{\sqrt{3}}{2} R a^{3}=457934 \AA^{3}$ (Tab. II). Knowing the density of the mixture and the molecular weight of $\mathrm{C}_{12} \mathrm{EO}_{8}\left(538 \mathrm{~g} \mathrm{~mol}^{-1}\right)$, we derive $N_{\mathrm{S}}=207$, the number of surfactant molecules per unit cell. With two micelles per unit cell in the structure, we get an aggregation number $N=103$.

A similar calculation is performed for the sample of concentration $40 \%$, the one which exhibits a cubic micellar phase (Tab. I). We find a number of surfactant molecules per unit cell: $N_{\mathrm{S}}=$ 824. The structure contains height micelles per unit cell, but with two different types of shape, so we can derive only an average aggregation number $\langle N\rangle=103$.

A neutron scattering study [8] estimate the aggregation number of the direct micelles in the isotropic phase. This study has been performed for temperatures between 30 and $60{ }^{\circ} \mathrm{C}$ and for weight concentrations of $\mathrm{C}_{12} \mathrm{EO}_{8}$ in $\mathrm{H}_{2} \mathrm{O}$ between $2.5 \%$ and $35 \%$. Aggregation numbers are found between 90 and 140; they increase versus the concentration or versus the temperature. In this region of the phase diagram, the micelles are expected to be quite spherical.

In short, we can say that an aggregation number of 103 is convenient for quasi-spherical micelles formed by $\mathrm{C}_{12} \mathrm{EO}_{8}$ molecules. The models we propose with two micelles per unit cell for the hexagonal phase, and height micelles per unit cell for the cubic phase appear to be consistent with the physico-chemical data. We also find that the average aggregation number is quite similar for both micellar phases.

\section{Discussion}

Thanks to the X-ray scattering analysis of single-crystals (3.3), we have identified a new type of micellar phase. We suggest it has a hexagonal compact structure with two micelles per unit cell (space group $\mathrm{P}_{3} / \mathrm{mmc}$ ). This model is consistent with the aggregation number of quasi-spherical micelles (3.4).

There remains a puzzling question about this phase: the optical observation of the samples between crossed polarizers does not reveal any birefringence (3.1), as expected in the case of a hexagonal symmetry. This implies that the anisotropy of the dielectric tensor is very weak, and that only appropriate measurements of birefringence could be able to detect it. Of course, such a measure would be a confirmation of the hexagonal symmetry.

We also notice that the ratio $R=c / a$ of the hexagonal unit cell (Tab. II) is very close to the value corresponding to a hexagonal close compact packing of spheres: $R_{\text {hcc }}=\sqrt{8 / 3}=1.6330$. In our opinion, it means that the micelles are rather quasi-spherical than anisotropic. 
To conclude, we see that the $\operatorname{Pm} \overline{3} \mathrm{n}$ and $\mathrm{Fd} \overline{3} \mathrm{~m}$ cubic structures are not the only solutions for direct micellar arrangements. The ganglioside/water systems [4] and now the $\mathrm{C}_{12} \mathrm{EO}_{8} /$ water binary system are two examples of different arrangements. They have several common features: a single type of micelle (instead of two types of shape in coexistence), having a quasi-spherical shape. All space groups correspond to compact arrangements of spheres: $\mathrm{P} 6_{3} / \mathrm{mmc}$ for the hexagonal phase, Fm $3 \mathrm{~m}$ and $\operatorname{Im} 3 \mathrm{~m}$ in the case of [4], respectively the closest and second closest cubic packing of spheres. The nature of a micellar phase is obviously related to the shape of the micelles: quasi-spherical or ellipsoidal.

\section{Acknowledgments}

We are very grateful to A.M. Levelut and J. F. Sadoc (Laboratoire de Physique des Solides, Orsay) for their constant encouragement during this work.

\section{References}

[1] Luzzati V., Vargas R., Mariani P., Gulik A. and Delacroix H., J. Mol. Biol. 229 (1993) 540.

[2] Fontell K., Collö̈d Polym. Sci. 268 (1990) 264.

[3] Charvolin J. and Sadoc J.F.. J. Phys. France 49 (1988) 521.

[4] Gulik A., Delacroix H., Kirschner G. and Luzzati V., J. Phys. II France 5 (1995) 445.

[5] Israelachvili J. N., Mitchell J. and Ninham B. W., J. Chem. Soc. Faraday Trans. II 72 (1976) 1523-1568.

[6] Richtering H. W., Burchard W., Jahns E. and Finkellmann H., J. Phys. Chem. 92 (1988) 6032.

[7] Nikko Chemicals Company. 1, 4, 8 Nihonbashi-Badurocko. Chuoko, Tokyo 103, Japan.

[8] Zulauf M., Weckström K., Hayter J. B., Degiorgio V. and Corti M, J. Phys. Chem. 89 (1985) 3411. 